\title{
Added value of spectral parameters for the assessment of lymph node metastasis of lung cancer with dual-layer spectral detector computed tomography
}

\author{
Lu Gao ${ }^{1}$, Xiaomei Lu², Qingyun Wen ${ }^{1}$, Yang Hou ${ }^{1}$ \\ ${ }^{1}$ Department of Radiology, Shengjing Hospital of China Medical University, Shenyang, China; ${ }^{2}$ CT Clinical Science, Philips Healthcare, Shenyang, \\ China
}

Correspondence to: Yang Hou. Department of Radiology, Shenging Hospital of China Medical University, No. 36, Sanhao Street, Shenyang 110004, China. Email: houyang1973@163.com.

Background: Lymph node (LN) metastasis is an important factor affecting the treatment of lung cancer. The purpose of this article was to investigate the benefits of dual-layer spectral detector computed tomography (SDCT) for the evaluation of metastatic LNs in lung cancer.

Methods: Data from 93 patients with lung cancer who underwent dual-phase enhanced scanning with SDCT were retrospectively analyzed. According to the pathological findings, 166 LNs were grouped as metastatic $(n=80)$ or non-metastatic $(n=86)$. LNs in station $4(n=80)$ and station $7(n=35)$ accounted for the majority of the LNs (approximately 69.23\%). The short-axis diameter of the LN, arterial enhancement fraction (AEF), normalized iodine concentration (NIC), and the slope of the spectral Hounsfield unit curve $\left(\lambda_{\mathrm{HU}}\right)$ during the arterial phase (AP) and venous phase (VP) were measured. The Mann-Whitney U test was used to statistically compare these quantitative parameters. Receiver operating characteristic (ROC) curves were plotted to identify the cutoff values, and decision curve analysis (DCA) was performed to determine the net benefit of each parameter. The diagnostic performance, obtained by combining the short-axis diameter with each of the above parameters, was also studied.

Results: The short-axis LN diameter, AEF, NIC, and $\lambda_{\text {HU }}$ during the AP and VP all showed significant differences between the metastatic and non-metastatic groups $(\mathrm{P}<0.05)$. Of the parameters, the AEF had the greatest diagnostic efficiency for metastatic LNs [area under the ROC curve (AUC) $)_{\mathrm{AEF}}=0.885$ ] with a threshold of $86.40 \%$. The sensitivity, specificity, positive predictive value (PPV), negative predictive value (NPV), and 95\% confidence interval were 90.00\%, 89.53\%, 88.89\%, 90.59\%, and 0.830-0.944, respectively. When the quantitative parameters were combined with the short-axis diameter, the AUCs of the parameters, except the AEF, were significantly improved $(\mathrm{P}<0.05)$.

Conclusions: The iodine quantitative parameters from SDCT, such as the AEF, demonstrated high diagnostic performances in the differentiation of metastatic and non-metastatic LNs.

Keywords: Tomography; X-ray computed; spectral CT; lymph nodes (LNs); lung cancer

Submitted Sep 08, 2020. Accepted for publication Feb 06, 2021.

doi: 10.21037 /qims-20-1045

View this article at: http://dx.doi.org/10.21037/qims-20-1045 


\section{Introduction}

Lung cancer is the leading cause of cancer-associated death globally (1). The clinical stage of lung cancer is an important factor affecting treatment (2). In addition to tumor size and distant metastasis, metastasis to hilar and mediastinal lymph nodes (LNs) is key to determining the lung cancer stage. Clinically, the conventional method for judging whether lung cancer has metastasized to LNs is based on measurement of the short-axis diameter of LNs on computed tomography (CT). However, when a short LN diameter of $>10 \mathrm{~mm}$ is used as the criterion for judging $\mathrm{LN}$ metastasis, the median sensitivity and specificity were $55 \%$ and $81 \%$, respectively (3). Therefore, an imaging method that can accurately assess LNs in patients with lung cancer is desirable.

Dual-energy CT (DECT) can show the regional perfusion state of normal and abnormal tissues through the quantification of iodine concentration (IC) $(4,5)$. So far, various studies have investigated the application value of DECT quantitative parameters for evaluating metastatic LNs in lung cancer (6-9). For instance, in a study of 144 LNs, Yang et al. (6) showed a good diagnostic performance using the slope of the spectral Hounsfield unit curve $\left(\lambda_{\mathrm{HU}}\right)$ [area under the receiver-operating curve $(\mathrm{AUC})_{\mathrm{AP}} \lambda_{\mathrm{HU}}$ $=0.951]$ and normalized iodine concentration (NIC) $\left(\mathrm{AUC}_{\mathrm{AP}}\right.$ NIC $=0.833)$. Meanwhile, Li et al.'s study of 40 LNs showed a moderate performance using NIC $\left(\mathrm{AUC}_{\mathrm{AP} \mathrm{NIC}}=0.700\right)(7)$. These inconsistencies in diagnostic performance could be attributable to numerous factors, such as differences in equipment, scanning parameters, sample size, and pathological type. Therefore, the diagnostic value of various quantitative parameters using DECT needs to be further explored.

Spectral detector CT (SDCT) uses a single X-ray source and a dual-layer detector, which absorbs photons of lower energies in the upper layer and photons of higher energies in the lower layer (10). The simultaneous, homologous, and co-directional characteristics of SDCT yield better data registration and image correspondence in different phases compared to DECT, which reduces measurement errors (11). The application of anti-correlated noise significantly reduces image noise, which makes the image clearer. Furthermore, the spectral base image (SBI) datasets reconstructed with an iterative reconstruction (IR) algorithm contain relevant information for retrospective analysis and eliminate the need for further reconstruction or post-processing (12). To date, only image quality and iodine attenuation have been used to investigate chest disease using SDCT $(13,14)$, and the incremental value of spectral parameters in the differential diagnosis of LN metastasis of lung cancer has not yet been reported. Therefore, the purpose of this study was to investigate the added value of spectral quantitative parameters for the evaluation of $\mathrm{LN}$ metastasis of lung cancer using SDCT.

\section{Methods}

\section{Study population}

This retrospective research was approved by the institutional review board of Shengjing Hospital of China Medical University (Shenyang, China). This study randomly selected 131 patients who underwent SDCT scanning and were given a primary imaging diagnosis of lung cancer between December 2017 and January 2019.

The inclusion criteria were as follows: (I) patients with enlarged mediastinal LNs with clear borders, and a short-axis diameter of the $\mathrm{LN}>5 \mathrm{~mm}$ in the axial image; (II) patients that had not undergone radiation therapy, chemotherapy, or targeted therapy for the tumor prior to the examination; (III) a pathological diagnosis of lung cancer and LNs diagnosed as non-metastatic or metastatic according to the post-surgical resection pathology report (including the location, size, number, shape of the tumor and LNs, as well as the presence or absence of metastasis); and (IV) comprehensive clinical data (inpatient medical records, changes in symptoms, blood tests, imaging reports, operation record, pathology reports) with good image quality (no artifacts) allowing for further analysis.

The exclusion criteria were as follows: (I) patients with lung cancer combined with other tumors; (II) patients with LNs that were fused together, which were not easy to measure and had multiple necrotic components, multiple calcifications (a calcified area larger than two-thirds of the area of the same layer of LNs); and (III) patients for whom the images and pathology report of the LNs did not match, including cases of location mismatch (the location displayed on the image was not consistent with the location marked in the pathology report), size mismatch (the size difference between the image and the pathological results was greater than $1 \mathrm{~mm}$ ), and number mismatch (the number of LNs displayed on the image in the same LN station was not consistent with the number recorded by the pathologist). 


\section{SDCT acquisition and post-processing}

All patients underwent dual-phase contrast-enhanced chest scans using SDCT (IQon Spectral CT, Philips Healthcare, Best, The Netherlands). The interval between the SDCT scan and the operation was usually several days, with the longest time for the included cases being 7 days. The reason for this is that, in our institution, it usually takes a few days for the clinician to comprehensively assess the patient's condition and determine the treatment method following the discovery of the tumor on SDCT. Shorter time intervals would strengthen the credibility of the results. Patients who were not suitable for surgical treatment after lung cancer staging or who were transferred to other institutions were not considered.

An in-dwelling trocar (Venflon) was placed in the elbow vein, and non-ionic iodine contrast agent iodixanol (iodicanol, $270 \mathrm{mg} \mathrm{I} / \mathrm{mL}$, GE Healthcare, Ireland) was injected at a volume of $80 \mathrm{~mL}$ and a rate of $2.5 \mathrm{~mL} / \mathrm{s}$ using a high-pressure injector (Ulrich REF XD 2051, Ulrich $\mathrm{GmbH} \& \mathrm{Co}$. KG, Germany). Arterial and venous images were acquired at 25 seconds and 60 seconds after the injection, respectively. The acquisition parameters were as follows: tube voltage $120 \mathrm{kVp}$; tube current modulation with the dose right index (DRI) set to 22; rotation speed $0.33 \mathrm{sec} / \mathrm{rot}$; helical pitch 0.671 ; detector collimation $64 \times 0.625 \mathrm{~mm}$; image reconstruction matrix $512 \times 512$. The data images were reconstructed as SBI datasets, with the spectral reconstruction level set to 3 , a reconstructed slice thickness of $1 \mathrm{~mm}$, and an increment of $1 \mathrm{~mm}$. Images reconstructed with the standard kernel (B) were reviewed in a mediastinal window with a width of 350 and a level of 60 . All images were then transferred to a spectral CT-dedicated post-processing workstation (IntelliSpace Portal Version 6.5, Philips Healthcare, Best, The Netherlands) for further analysis.

\section{Data analysis}

According to the LN map of lung cancer proposed by the International Association for the study of Lung Cancer (IASLC) (15), 2 chest radiologists with more than 10 years of experience independently analyzed the quantitative parameters. Both radiologists were blinded to the pathological results. In cases of dispute $(\mathrm{n}=26$, including 5 valid LNs), an agreement was reached through consultation. The reason for such disagreements was the $\mathrm{LN}$ having a deep location, which made measurement difficult. For instance, LNs located at $10^{\text {th }}$ (hilus of the lung) and $13^{\text {th }}$ (lung segment) were sometimes indistinguishable from the surrounding lung tissues or blood vessels; therefore, we usually chose LNs with clear boundaries and higher enhancement than the surrounding tissues.

In the $70 \mathrm{keV}$ image, the largest section of the solid component of the target $\mathrm{LN}$, and the upper and lower layers in the axial image were selected as regions of interest (ROIs). The average value was taken, and the average of 2 independent measurement results was calculated and used as the final result. ROIs were circular in shape, and the copy-and-paste function was used to ensure the size, shape, and position of all ROIs were identical. ROIs were drawn to avoid the edges of LNs and internal blood vessels, as well as any calcification and necrosis as much as possible. Also, to avoid selection bias, we selected only 1 station area with 1 pathological type of LN (metastatic/non-metastatic), and there were fewer than $3 \mathrm{LNs}$ in the same area.

The short-axis diameter of the target $\mathrm{LN}$ was measured in the $70 \mathrm{keV}$ image. The SDCT post-processing workstation automatically calculates the IC within the ROI in the iodine-based material image. Selecting the same layer of aortic IC to standardize the measured LNs can help to avoid the effects of inter-individual differences. The formula of the related spectral parameters is as follows: $\mathrm{NIC}=\mathrm{IC}_{\mathrm{LN}} / \mathrm{IC}_{\text {aorta }}(6)$. Arterial enhancement fraction (AEF) $=$ iodine uptake in the arterial phase (AP)/iodine uptake in venous phase $(\mathrm{VP}) \times 100 \%(8,9)$. For energy levels greater than $120 \mathrm{keV}$, the spectral curve exhibited smaller changes and differences than those that occurred below $120 \mathrm{keV}$ (16). Therefore, in this study, the $40-80 \mathrm{keV}$ energy range was selected for analysis, which corresponds to $\lambda_{\mathrm{HU}}=\left(\mathrm{CT}_{40 \mathrm{keV}}-\right.$ $\left.\mathrm{CT}_{80 \mathrm{keV}}\right) / 40$.

\section{Statistical analysis}

SPSS 20.0 (SPSS, Inc., Chicago, IL, USA) and MedCalc15.6 (MedCalc Software, Mariakerke, Belgium) were used for all statistical analyses. The KolmogorovSmirnov test was used to determine the normality of the data. Moreover, except for VP NIC, the parameters did not fit a normal distribution. The data were provided as the mean \pm standard deviation (SD) for normally distributed data, and as the median $\left(25^{\text {th }}-75^{\text {th }}\right.$ percentiles $)$ for nonnormally distributed data.

Since this study involved the cases of patients with multiple LNs, all quantitative parameters were tested using the Mann-Whitney U test. Based on the receiver operating 


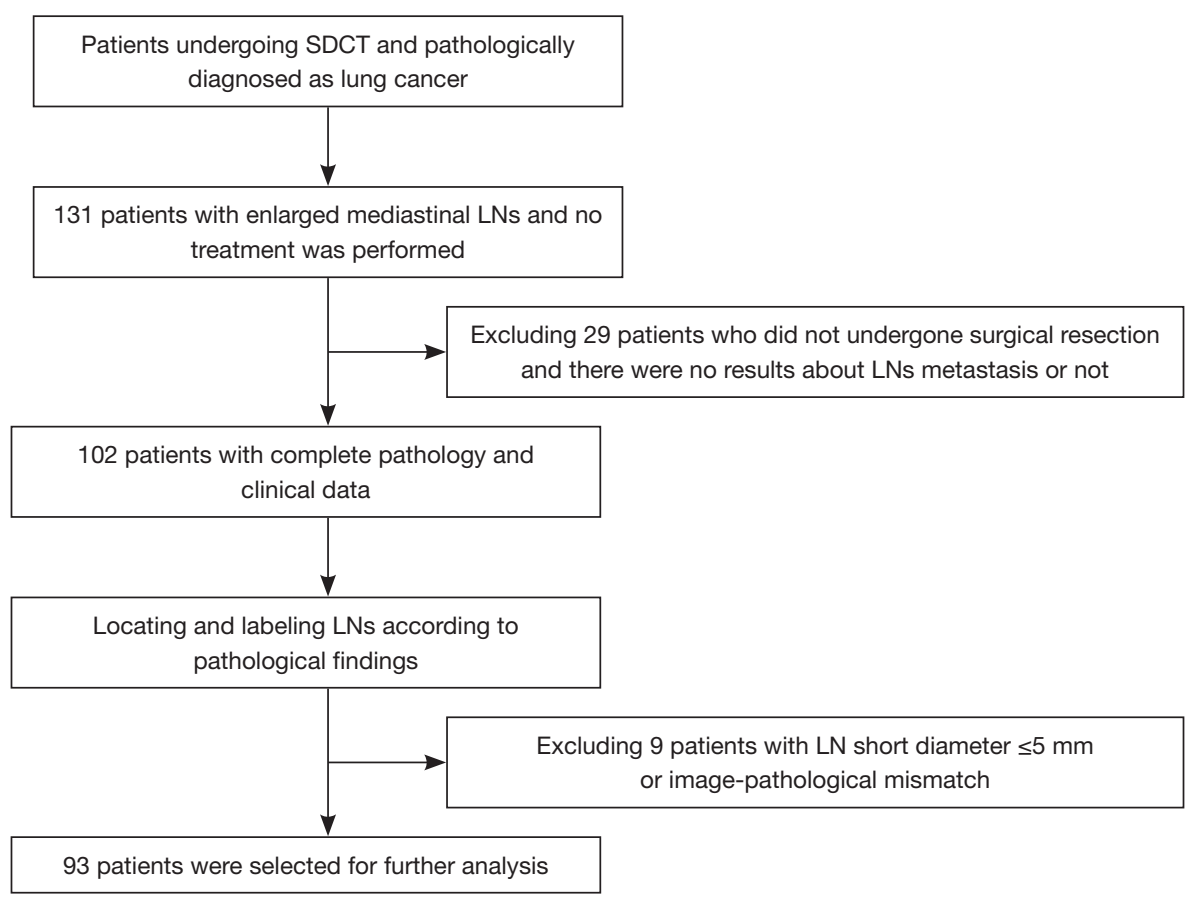

Figure 1 Flow diagram of patient selection. LN, lymph node; SDCT, spectral detector computed tomography.

characteristic (ROC) curve, the cutoff value was obtained to identify whether the lung cancer had metastasized to LNs. At the same time, the diagnostic capability of each dual-energy spectral quantitative parameter was compared through the AUC. After the cutoff value had been determined, the sensitivity, specificity, positive predictive value (PPV), and negative predictive value (NPV) of each quantitative parameter were calculated. The spectral parameters and the short-axis diameters of the LNs were combined, and logistic regression analysis was performed, and then the difference between the parameters before and after combination was analyzed. A value of $\mathrm{P}<0.05$ was considered to be statistically significant. Decision curve analysis (DCA) was also performed to determine the net benefit of each individual and combined parameter.

\section{Results}

\section{Patients and LNs}

All patients underwent contrast-enhanced chest enhancement CT examination, and no adverse reactions were reported. A total of 93 patients were included in the study $(57$ men and 36 women; age, $61.18 \pm 9.00$ years; 42 metastatic cases, 51 non-metastatic cases). Figure 1 displays the process of patient selection. A total of 166 valid LNs were confirmed by pathology and were grouped as metastatic $(\mathrm{n}=80)$ or nonmetastatic $(n=86)$. Of them, LNs in stations $4(n=80)$ and 7 $(\mathrm{n}=35)$ accounted for the majority of LNs (approximately 69.23\%). Patient characteristics and lesion types are shown in Table 1. The distributions of LNs in each group are shown in Figure 2.

\section{Size of $L N$}

In the $70 \mathrm{keV}$ images, the diameter of the short axis was $10.20(7.93-13.10) \mathrm{mm}$ and 8.10 (6.93-9.60) $\mathrm{mm}$ (Table 2) for the metastatic and non-metastatic groups, respectively. Compared with the accepted diagnostic criteria of LN short-axis diameter $>10 \mathrm{~mm}$ (3), the cutoff value determined in the current study was $9.70 \mathrm{~mm}$, for which the AUC was 0.680 , and the sensitivity, specificity, PPV, NPV, and accuracy were $53.75 \%, 77.91 \%, 69.35 \%, 64.42 \%$, and $66.27 \%$, respectively, for identifying LN metastasis (Table 3).

\section{Comparison of the individual SDCT quantitative parameters in the diagnosis of metastatic LNs}

The AEF, $\lambda_{\mathrm{HU}}$, and NIC of metastatic and non-metastatic LNs measured during the AP and VP were statistically different $(\mathrm{P}<0.05)$ (Table 2). The AUC, sensitivity, 
Table 1 Patient characteristics $(n=93)$

\begin{tabular}{lc}
\hline Clinical factors & No. $(\%)$ \\
\hline Sex & $57(61.3)$ \\
Male & $36(38.7)$ \\
Female & \\
Age (years) & $10(10.8)$ \\
$<50$ & $36(38.7)$ \\
$50-60$ & $33(35.5)$ \\
$60-70$ & $14(15.0)$ \\
$>70$ & \\
Pathologic type & $49(52.7)$ \\
Adenocarcinoma & $19(20.4)$ \\
Metastasis & $30(32.3)$ \\
No metastasis & $22(23.7)$ \\
Squamous cell carcinoma & $7(7.5)$ \\
Metastasis & $15(16.1)$ \\
No metastasis & $22(23.7)$ \\
Small cell carcinoma & $19(20.4)$ \\
Metastasis & $3(3.2)$ \\
No metastasis & \\
\hline
\end{tabular}

specificity, PPV, NPV, and cutoff value of each quantitative parameter for the 2 groups are shown in Table 3. There was a statistical difference in the AUC of each parameter $(\mathrm{P}<0.05)$. The AEF had the highest AUC $(\mathrm{AUC}=0.885)$ of all the quantitative parameters $\left(\mathrm{P}_{\mathrm{AEF}-\mathrm{AP} \mathrm{NIC}}=0.048, \mathrm{P}_{\mathrm{AEF}}\right.$ AP $\lambda \mathrm{HU}=0.042, \mathrm{P}_{\mathrm{AEF}-\mathrm{VP} N \mathrm{NIC}}<0.001, \mathrm{P}_{\mathrm{AEF}-\mathrm{VP}} \lambda \mathrm{HU}<0.001$, and $\mathrm{P}_{\mathrm{AEF}-}$ Size $<0.001$ ) (Figures 3 and 4 ), as well as the largest net benefit (Figure 3). With a cutoff value of $86.40 \%$, the diagnostic sensitivity, specificity, PPV, and NPV of the AEF were $90.00 \%, 89.53 \%, 88.89 \%$, and $90.59 \%$, respectively. Furthermore, among the 9 false positive LNs, there were 7 adenocarcinomas (4 of which were poorly differentiated) and 2 moderately or poorly differentiated squamous cell carcinomas. Among the 8 false negative LNs, there were 5 small cell carcinomas, and 3 moderately or poorly differentiated adenocarcinomas.

\section{Diagnostic value of the combination of SDCT quantitative parameters and the short-axis diameter}

The morphological features of the short-axis diameter of the LNs were combined with the SDCT quantitative parameters, and a logistic regression analysis was performed (Table 4). The combined ROC curves and DCA are shown in Figure 3. After the combination of quantitative parameters with the short-axis diameter, the AUC of the combination of VP NIC and the short-axis diameter showed the highest increase $(9.53 \%)$, while the combination of the AEF and the short-axis diameter had the largest net benefit (Figure 3). However, the degree of improvement was not statistically different compared to the AEF alone $\left(\mathrm{AUC}_{\mathrm{AEF}}=0.885 \& \mathrm{AUC}_{\mathrm{AEF}+\mathrm{Size}}=0.889\right)$. The other parameters showed differences between their performances alone and in combination with the short-axis diameter $\left(\mathrm{P}_{\mathrm{AP}}\right.$ NIC-AP NIC+Size $=0.017, \mathrm{P}_{\mathrm{VP}}$ NIC-VP NIC+Size $=0.041, \mathrm{P}_{\mathrm{AP}} \lambda \mathrm{HU}_{-\mathrm{AP}} \lambda \mathrm{HU}_{+}$Size $=0.036$, and $\left.\mathrm{P}_{\mathrm{VP} \lambda \mathrm{HU}-\mathrm{VP} \lambda \mathrm{HU}+\text { Size }}=0.046\right)$. Furthermore, after combination, among the 10 false positive LNs, there were 6 adenocarcinomas (4 of which were poorly differentiated), 3 poorly differentiated squamous cell carcinomas, and 1 small cell lung cancer. Among the 7 false negative LNs, there were 4 small cell carcinomas, and 3 moderately or poorly differentiated adenocarcinomas.

\section{Discussion}

This study showed that multiple quantitative iodine parameters in SDCT had a better diagnostic performance than the short-axis diameter alone in the assessment of $\mathrm{LN}$ metastasis of lung cancer. Of the parameters, the AEF had the highest diagnostic efficacy, with an accuracy of $89.76 \%$.

To our knowledge, this is the first study to evaluate LN metastasis using quantitative parameters obtained with SDCT. The metastasis of LNs is an important factor affecting the staging of lung cancer $(2,17)$. The use of a single anatomical measurement alone, such as the short-axis diameter of $\mathrm{LN}$, has certain limitations in the identification of metastatic LNs (17). This was confirmed in our study, which further demonstrated that the spectral parameters of the AP had a better diagnostic performance than the shortaxis diameter alone. This may be related to the quantitative parameters of the energy spectrum and can better reflect the pathological characteristics of metastatic LNs.

Tumor cells release regulatory factors to stimulate microvascular regeneration in LNs before metastasis occurs $(18,19)$. Malignant LNs have a rich blood supply compared to benign LNs (20). The large number of micro-vessels, relatively immature blood vessels, and the high density of micro-vessels. increase the accumulation of contrast agent in metastatic LNs $(21,22)$, which is exhibited through rapid 


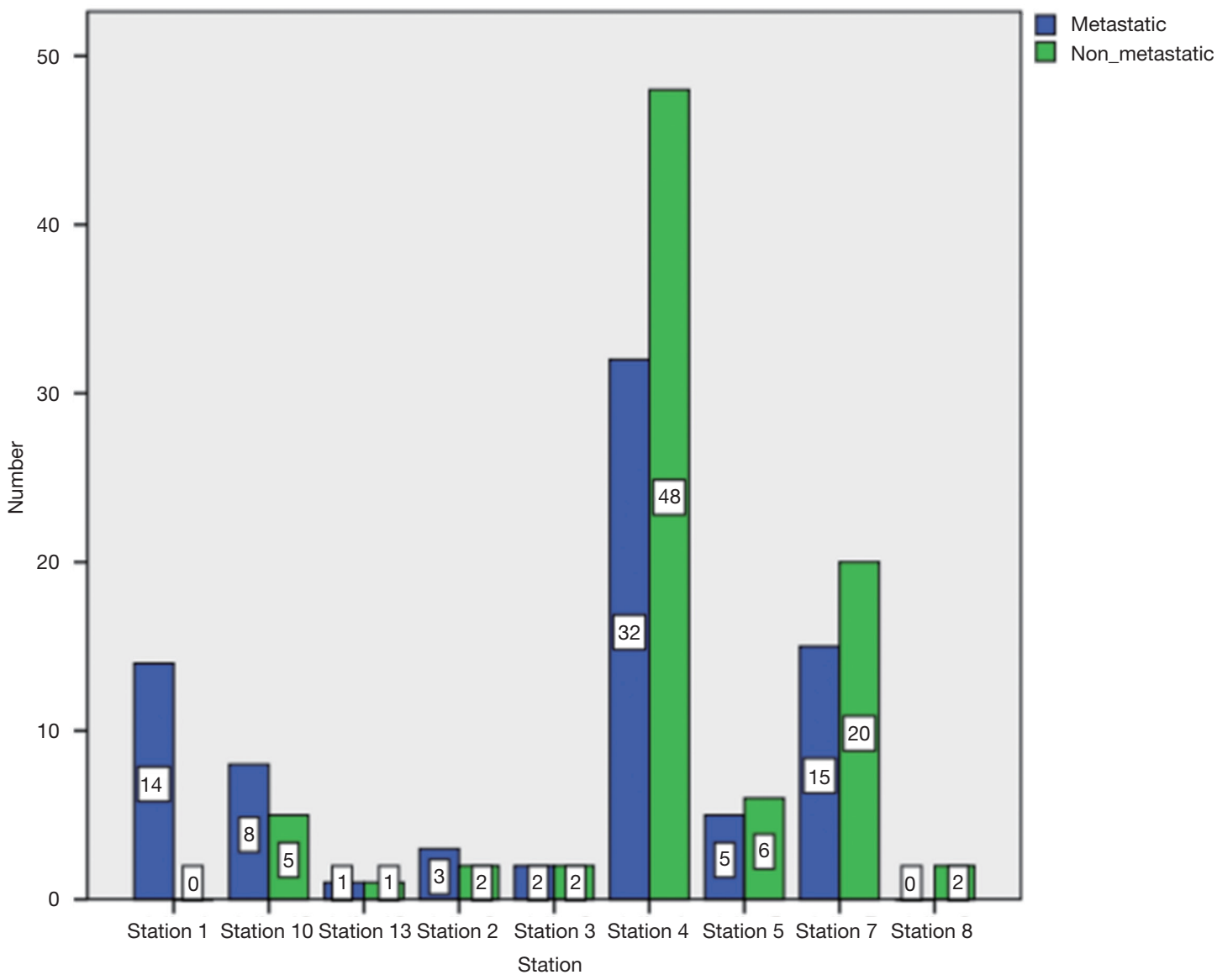

Figure 2 Distribution of LNs (80 metastatic LNs and 86 non-metastatic LNs). LN, lymph node.

Table 2 Differences in SDCT quantitative parameters between metastatic and non-metastatic LNs

\begin{tabular}{lccc}
\hline Parameters & Metastatic group & Non-metastatic group & P value \\
\hline AEF & $95.10(89.00-112.15)$ & $77.78(69.23-83.15)$ & 0.000 \\
AP NIC & $0.18(0.14-0.22)$ & $0.13(0.10-0.17)$ & 0.000 \\
VP NIC & $0.38 \pm 0.08$ & $0.32 \pm 0.09$ & 0.001 \\
AP $\lambda_{H U}$ & $2.45(1.73-3.18)$ & $2.05(1.30-2.79)$ & 0.000 \\
VP $\lambda_{H U}$ & $2.67(2.08-3.01)$ & $2.30(1.96-2.71)$ & 0.000 \\
Short-axis diameter & $10.20(7.93-13.10)$ & $8.10(6.93-9.60)$ & 0.000 \\
\hline
\end{tabular}

The unit of the short-axis diameter of the LNs was millimeter $(\mathrm{mm})$. Data are presented as mean \pm standard deviations or median $\left(25-75^{\text {th }}\right.$ percentiles). $\mathrm{P}<0.05$ indicates a statistically significant difference. SDCT, spectral detector computed tomography; AEF, arterial enhancement fraction; NIC, normalized iodine concentration; $\lambda \mathrm{HU}$, slope of the spectral Hounsfield unit curve; AP, arterial phase; VP, venous phase.

and intense contrast enhancement, showing a high iodine value on the iodine density map (23). This could help to explain the improved diagnostic performance with the use of spectral parameters compared to that of the short-axis diameter.
We found that the AEF had the highest diagnostic performance, as evidenced by the $\mathrm{AUC}\left(\mathrm{AUC}_{\mathrm{AEF}}=0.885\right)$, and the AEF value of metastatic LNs was higher than that of non-metastatic LNs. This is consistent with the findings of Baxa et al. (8), who used the AEF to identify metastatic 
Table 3 Diagnostic efficiency of SDCT quantitative parameters between metastatic and non-metastatic LNs

\begin{tabular}{|c|c|c|c|c|c|c|}
\hline Parameters & AEF & AP NIC & AP $\lambda_{H U}$ & VP NIC & VP $\lambda_{H U}$ & Size \\
\hline True positive (n) & 72 & 49 & 65 & 69 & 47 & 43 \\
\hline False negative (n) & 8 & 31 & 15 & 11 & 33 & 37 \\
\hline Sensitivity (\%) & 90 & 61.25 & 81.25 & 86.25 & 58.75 & 53.75 \\
\hline Specificity (\%) & 89.53 & 87.21 & 69.77 & 50 & 77.91 & 77.91 \\
\hline$+\mathrm{LR}$ & 8.59 & 2.77 & 3.12 & 2.1 & 5.38 & 2.58 \\
\hline$-\mathrm{LR}$ & 0.09 & 0.23 & 0.29 & 0.56 & 0.57 & 0.52 \\
\hline
\end{tabular}

${ }^{\dagger}, 95 \% \mathrm{Cl}$ means the $95 \%$ confidence interval, Size means the short-axis diameter of the LN. SDCT, spectral detector computed tomography; AUC, area under the ROC; AEF, arterial enhancement fraction; NIC, normalized iodine concentration; $\lambda_{H U}$, slope of the spectral Hounsfield unit curve; AP, arterial phase; VP, venous phase; PPV, positive predictive value; NPV, negative predictive value; +LR, positive likelihood ratio; -LR, negative likelihood ratio.

LNs in lung cancer $\left(\mathrm{AEF}_{\text {metastatic } \mathrm{LN}}=90.4 \%, \mathrm{AEF}_{\text {non-metastatic }}\right.$ $\mathrm{LNs}_{\mathrm{s}}=72.7 \%$ ). Based on this, their study further concluded that the diagnostic utility of the AEF was superior to that of other commonly used spectral indicators. In a recent investigation exploring the value of commonly used spectral parameters, Dong et al. (24) demonstrated that VP NIC (AUC $=0.87$ ) had a relatively good diagnostic performance; however, this parameter was shown to have moderate diagnostic capability by Li et al. $\left(\mathrm{AUC}_{\mathrm{AP} \text { NIC }}=0.700\right)(7)$. This is inconsistent with our findings. The reason why the diagnostic performance of the AEF is better than that of conventional parameters may be explained by the following. Firstly, in terms of hemodynamics, cancer cell infiltration induces angiogenesis in LNs (25). Increased angiogenesis is accompanied by an increased distribution of contrast agents in the intravascular/extravascular compartment (8), thereby resulting in a higher AEF value during the same circulatory phase. Additionally, unlike NIC and $\lambda_{\mathrm{HU}}$, the AEF can distinguish the relationship between different phases of the same blood circulation (26). Secondly, the spectral detector CT used in this study facilitates the simultaneous acquisition of low- and high-energy data at the same spatial and temporal position (27), which provides perfectly aligned data with significantly reduced measurement error, with no a priori decision needed for the mode of acquisition (27). These reasons may account for the superior performance of AEF in our study.

In this study, each of the quantitative parameters was further combined with the short-axis diameter of the LNs, and the diagnostic performance of the combined parameters was improved (except for that of the AEF), although the AEF still had the highest diagnostic efficiency. Similar improvements in performance using spectral combinations were reported by Rao et al. (28). As in this study, the authors reported improvements in sensitivity (75$87.5 \%)$ and specificity (73-96.2\%) with the combination of NIC and short-axis diameter through sequential or parallel tests. Our study also showed improvements in the diagnostic performance of each of the spectral parameters after combination with the short-axis diameter. However, before and after combination, adenocarcinoma accounted for a large proportion of false positive LNs $(7 / 9,77.78 \%$ and $6 / 10,60.00 \%$, respectively) and small cell carcinomas accounted for a large proportion of false negative LNs (5/8, $62.50 \%$ and $4 / 7,57.14 \%$, respectively). This could be explained by the highly aggressive nature of small cell 
A

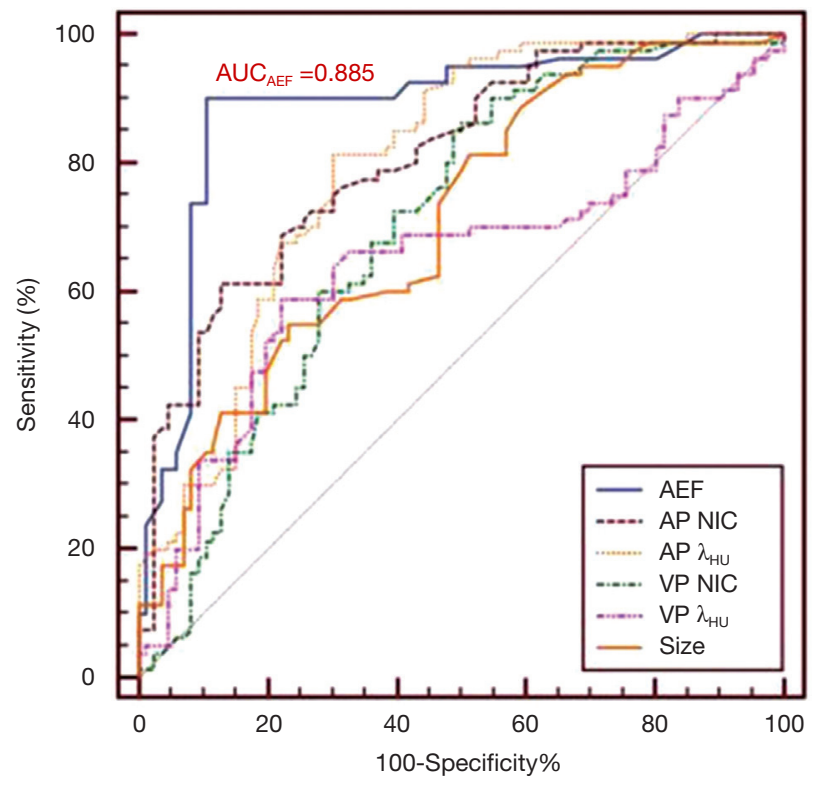

C

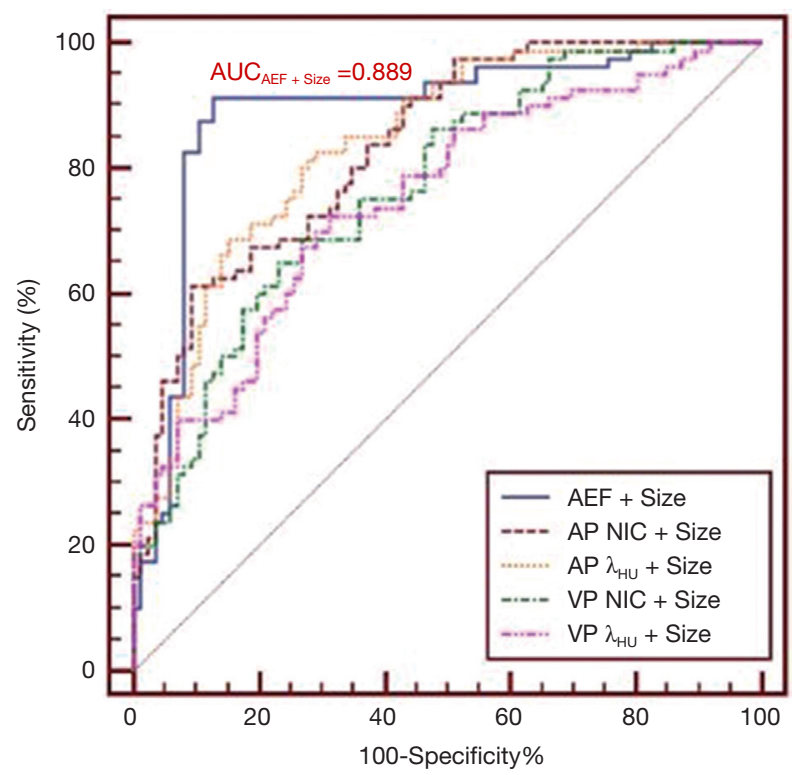

B

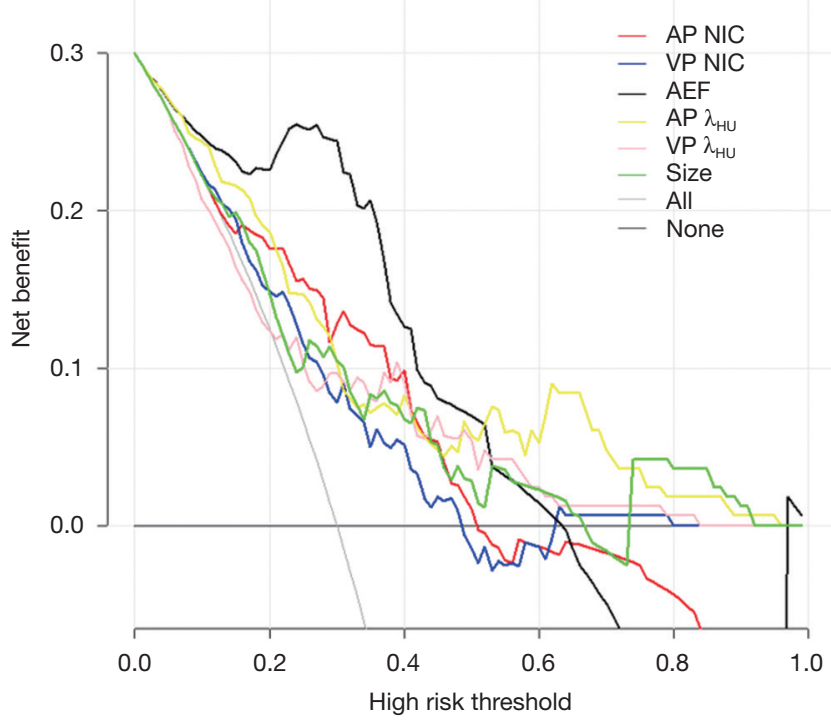

D

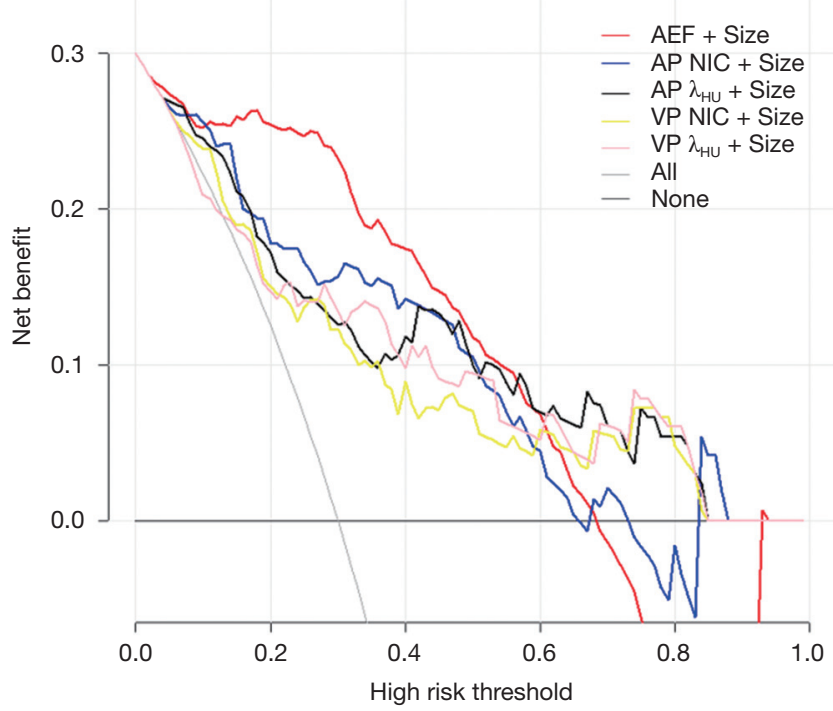

Figure 3 The ROC curves and decision curve analysis (DCA) for individual SDCT quantitative parameters and the combination with shortaxis diameter in the diagnosis of metastatic LNs. (A) ROC curves for SDCT quantitative parameters to distinguish metastatic LNs. The AEF had the highest diagnostic efficiency with an AUC of 0.885 (95\% CI: 0.830-0.941). (B) Decision curve analysis (DCA) of SDCT quantitative parameters. (C) ROC curves for the combinations of SDCT quantitative parameters with the short-axis diameter of the LN. (D) DCA of combined parameters of SDCT quantitative parameters with the short-axis diameter of the LN. AEF, arterial enhancement fraction; ROC, Receiver operating characteristic; SDCT, spectral detector computed tomography; LN, lymph node; NIC, normalized iodine concentration.

carcinoma (29) and the large amount of cell necrosis (30). It is also related to the iodine uptake in low-grade nonsmall cell lung cancer (NSCLC) being higher than that in high-grade NSCLC (25). Finally, considering that sample selection deviation may cause a high positive rate of metastatic LNs, which in turn leads to an overestimation of 

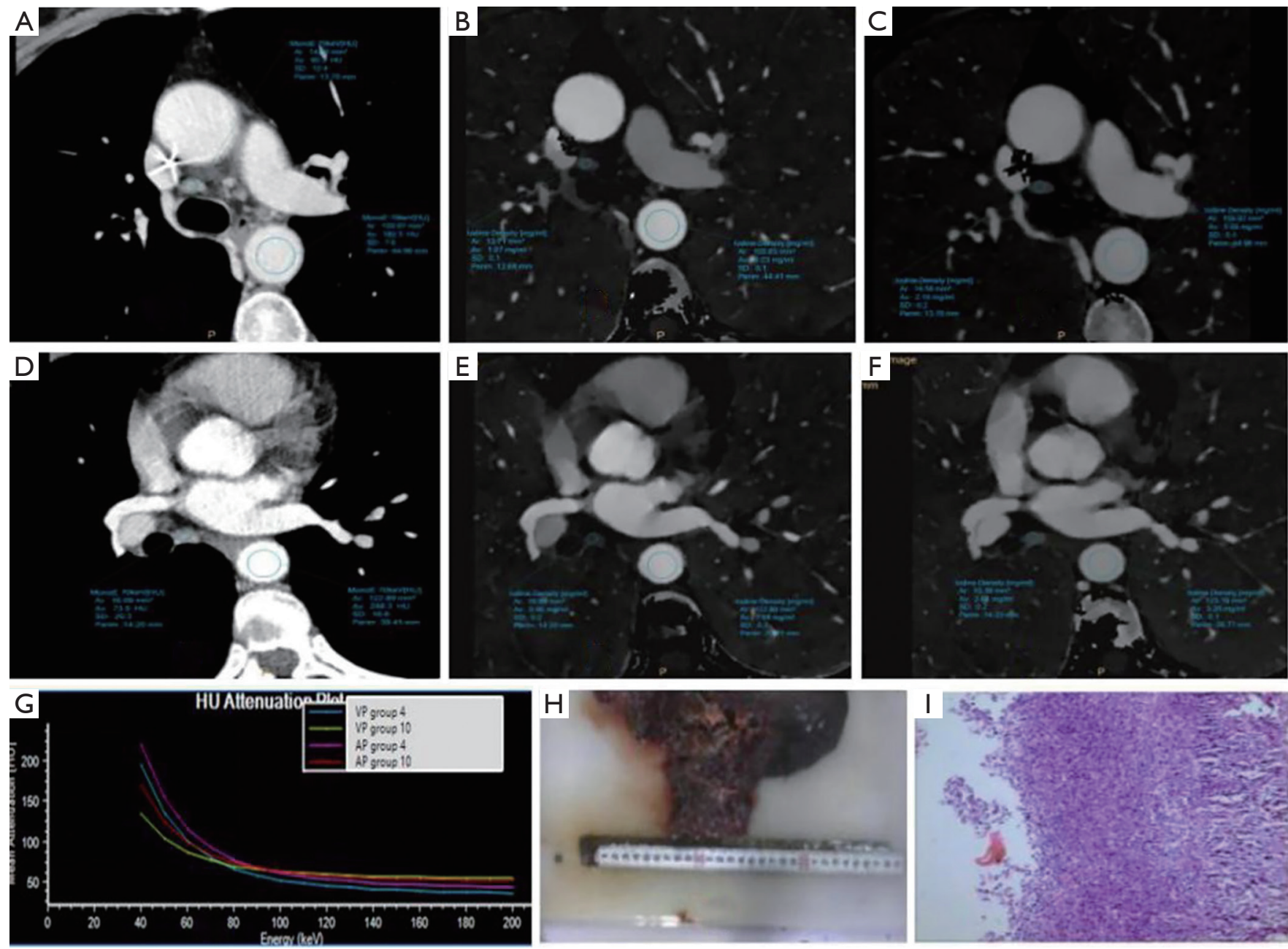

Figure 4 CT images of a 67-year-old male patient with adenocarcinoma. The 70-keV monochromatic image in the mediastinal window during the AP (A,D). Iodine density image in the same layer in the AP (B,E) and VP (C,F). Spectral curves in the AP and VP (G). Pathological diagnosis was adenocarcinoma (H,I; I, magnification, $\times 10$; HE staining). LNs in group 4 were metastatic and LNs in group 10 were non-metastatic. For group 4 LNs: short-axis diameter: $0.56 \mathrm{~cm}$, AEF: $89.54 \%$, AP NIC: 0.24 , VP NIC: 0.40 , AP $\lambda_{\text {HU }} 3.13$, VP $\lambda_{\text {HU: }}$ 2.49. Of these, AEF, AP NIC, VP NIC and AP $\lambda_{\mathrm{HU}}$, and VP $\lambda_{\mathrm{HU}}$ can correctly diagnose the metastatic LNs in group 4 . For group 10 LNs: short-axis diameter: $0.64 \mathrm{~cm}$, AEF: 47.76\%, AP NIC: 0.12, VP NIC: 0.38 , AP $\lambda_{\mathrm{HU}}: 1.88$, and VP $\lambda_{\mathrm{HU}}: 1.08$. Of these, AEF, AP NIC, AP $\lambda_{\mathrm{HU}}$, and VP $\lambda_{\mathrm{HU}}$ can all correctly diagnose the non-metastatic LNs in group 10. AP, arterial phase; VP, venous phase; AEF, arterial enhancement fraction; NIC, normalized iodine concentration.

the research results, this study used a likelihood ratio (LR) to evaluate the credibility of the results. It was obtained from the results that a single AEF has a greater diagnostic performance in judging LN metastasis (+LR, 8.59; -LR, 0.09) than other parameters, but when combined with the short-axis diameter of LNs, the diagnostic ability decreases (+LR, 5.12; -LR, 0.10). However, when other parameters are combined with the short-axis diameter, the credibility of LN diagnosis increases.
There are some limitations in this study that should be noted. Firstly, this was an exploratory study, and the sample size was small. Therefore, the findings need to be further confirmed with larger-sample studies. Secondly, since the sample size was not large, and there were significant differences between the subgroups, this study did not further discuss the differences in spectral parameters between different pathological types, LN locations, ages and gender, which are worthy of exploration. Thirdly, this 
Table 4 Combination of SDCT quantitative parameters and the short-axis diameter of the LNs

\begin{tabular}{|c|c|c|c|c|c|}
\hline Combined parameters & AEF+Size & AP NIC+Size & 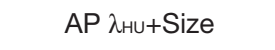 & VP NIC+Size & 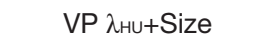 \\
\hline True positive (n) & 73 & 49 & 55 & 55 & 58 \\
\hline False positive (n) & 10 & 8 & 13 & 23 & 27 \\
\hline True negative $(\mathrm{n})$ & 76 & 78 & 73 & 63 & 59 \\
\hline Sensitivity (\%) & 91.25 & 61.25 & 68.75 & 68.75 & 72.5 \\
\hline Specificity (\%) & 88.37 & 90.7 & 84.88 & 73.26 & 68.6 \\
\hline PPV (\%) & 87.95 & 85.96 & 80.88 & 70.51 & 68.24 \\
\hline NPV (\%) & 91.57 & 71.56 & 74.49 & 71.59 & 72.83 \\
\hline$-\mathrm{LR}$ & 0.1 & 0.07 & 0.31 & 0.39 & 0.43 \\
\hline
\end{tabular}

${ }^{\dagger}$, 95\% Cl means the 95\% confidence interval, Size means the short-axis diameter of the LN. AUC, area under the ROC; AEF, arterial enhancement fraction; NIC, normalized iodine concentration; $\lambda_{H U}$, slope of the spectral Hounsfield unit curve; AP, arterial phase; VP, venous phase; PPV, positive predictive value; NPV, negative predictive value; +LR, positive likelihood ratio; -LR, negative likelihood ratio.

study only discussed the combination of 2 parameters at a time; the combination of multiple parameters may also be worth studying. Finally, in this study, 49 patients with more than $1 \mathrm{LN}$ and 3 patients with both metastatic and nonmetastatic LNs were included, which may have affected the results. To summarize, research on the added value of SDCT parameters in lung cancer staging is still in its early stages, and further exploration is still needed. In addition to the directions mentioned above, volumetric nodule measurements, the use of the AEF in the differentiation of benign and malignant lung masses, and the differences between different pathological types also warrant investigation.

\section{Conclusions}

The AEF is a better predictor of $\mathrm{LN}$ metastasis than the short-axis diameter of the $\mathrm{LN}$ in the evaluation of $\mathrm{LN}$ metastasis of lung cancer. Furthermore, the combination of spectral parameters with the short-axis diameter of LNs can help to distinguish LN metastasis.

\section{Acknowledgments}

We would like to thank Mani Vembar and Zheng Jia who assisted in the preparation of this paper.

Funding: This work was supported by Shengjing Hospital of China Medical University (345 Talent Project).

\section{Footnote}

Conflicts of Interest: All authors have completed the ICMJE uniform disclosure form (available at http://dx.doi. org/10.21037/qims-20-1045). The authors have no conflicts of interest to declare.

Ethical Statement: This retrospective research was approved by the institutional review board of Shengiing Hospital of China Medical University (Shenyang, China) and individual consent for this retrospective analysis was waived.

Open Access Statement: This is an Open Access article distributed in accordance with the Creative Commons Attribution-NonCommercial-NoDerivs 4.0 International License (CC BY-NC-ND 4.0), which permits the noncommercial replication and distribution of the article with the strict proviso that no changes or edits are made and the original work is properly cited (including links to both the formal publication through the relevant DOI and the license). See: https://creativecommons.org/licenses/by-nc-nd/4.0/. 


\section{References}

1. Siegel RL, Miller KD, Jemal A. Cancer statistics. CA Cancer J Clin 2018;68:7-30.

2. Rusch VW, Chansky K, Kindler HL, Nowak AK, Pass HI, Rice DC, Shemanski L, Galateau-Sallé F, McCaughan BC, Nakano T, Ruffini E, van Meerbeeck JP, Yoshimura M; IASLC Staging and Prognostic Factors Committee, advisory boards, and participating institutions. The IASLC Mesothelioma Staging Project: Proposals for the $\mathrm{m}$ descriptors and for revision of the TNM stage groupings in the forthcoming (eighth) edition of the TNM classification for mesothelioma. J Thorac Oncol 2016;11:2112-9.

3. Silvestri GA, Gonzalez AV, Jantz MA, Margolis ML, Gould MK, Tanoue LT, Harris LJ, Detterbeck FC. Methods for staging non-small cell lung cancer: Diagnosis and management of lung cancer, 3rd ed: American College of Chest Physicians evidence-based clinical practice guidelines. Chest 2013;143:E211-E250.

4. Chae EJ, Song JW, Seo JB, Krauss B, Song KS. Clinical utility of dual-energy CT in the evaluation of solitary nodules: Initial experience. Radiology 2008;249:671-81.

5. Chae EJ, Song JW, Krauss B, Song, KS, Lee CW, Lee HJ, Seo JB. Dual-energy computed tomography characterization of solitary pulmonary nodules. J Thorac Imaging 2010;25:301-10.

6. Yang F, Dong J, Wang X, Fu X, Zhang T. Non-Small cell lung cancer: Spectral computed tomography quantitative parameters for preoperative diagnosis of metastatic lymph nodes. Eur J Radiol 2017;89:129-35.

7. Li X, Meng X, Ye Z. Iodine quantification to characterize primary lesions, metastatic and non-metastatic lymph nodes in lung cancers by dual energy computed tomography: An initial experience. Eur J Radiol 2016;85:1219-23.

8. Baxa J, Vondráková A,Matouskova T, Ruzicková O, Schmidt B, Flohr T, Sedlmair M, Ferda J. Dual-phase dual-energy CT in patients with lung cancer: assessment of the additional value of iodine quantification in lymph node therapy response. Eur Radiol 2014;24:1981-8.

9. Baxa J, Matouskova T, Krakorova G, Schmidt B, Flohr T, Sedlmair M, Bejcek J, Ferda J. Dual-Phase Dual-Energy CT in Patients Treated with Erlotinib for Advanced NonSmall Cell Lung Cancer: Possible Benefits of Iodine Quantification in Response Assessment. Eur Radiol 2016;26:2828-36.

10. McCollough CH, Leng S, Yu L, Fletcher JG. Dual- and Multi-Energy CT: Principles, Technical Approaches, and
Clinical Applications. Radiology 2015;276:637-53.

11. Ananthakrishnan L, Rajiah P, Ahn R, Rassouli N, Xi Y, Soesbe TC, Lewis MA, Lenkinski RE, Leyendecker JR, Abbara S. Spectral detector CT-derived virtual noncontrast images: comparison of attenuation values with unenhanced CT. Abdom Radiol (NY) 2017;42:702-9.

12. Lu X, Lu Z, Yin J, Gao Y, Chen X, Guo Q. Effects of radiation dose levels and spectral iterative reconstruction levels on the accuracy of iodine quantification and virtual monochromatic CT numbers in dual-layer spectral detector CT: an iodine phantom study. Quant Imaging Med Surg 2019;9:188-200.

13. den Harder AM, Bangert F, van Hamersvelt RW, Leiner T, Milles J, Schilham AMR, Willemink MJ, de Jong PA. The effects of iodine attenuation on pulmonary nodule volumetry using novel Dual-Layer Computed Tomography Reconstructions. Eur Radiol 2017;27:5244-51.

14. Doerner J, Hauger M, Hickethier T, Byrtus J, Wybranski C, Hokamp NG, Maintz D, Haneder S. Image quality evaluation of dual-layer spectral detector CT of the chest and comparison with conventional CT imaging. Eur J Radiol 2017;93:52-8.

15. El-Sherief AH, Lau CT, Wu CC, Drake RL, Abbott GF, Rice TW. International Association for the Study of Lung Cancer (IASLC) Lymph Node Map: Radiologic Review with CT Illustration. Radiographics 2014;34:1680-91.

16. Wang Q, Shi G, Qi X, Fan X, Wang L. Quantitative analysis of the dual-energy CT virtual spectral curve for focal liver lesions characterization. Eur J Radiol 2014;83:1759-64.

17. Walker CM, Chung JH, Abbott GF, Little BP, ElSherief AH, Shepard JAO, Lanuti M. Mediastinal lymph node staging: From noninvasive to surgical. AJR Am J Roentgenol 2012;199:W54-64.

18. Lee SY, Chao-Nan Q, Seng OA, Peiyi C, Bernice WHM, Swe WS, Chii WJ, Jacqueline HSG, Chee SK. Changes in specialized blood vessels in lymph nodes and their role in cancer metastasis. J Transl Med 2012;10:206.

19. Zhao Y, Li X, Li L, Wang X, Lin M, Zhao X, Luo D, Li J. Preliminary study on the diagnostic value of singlesource dual-energy CT in diagnosing cervical lymph node metastasis of thyroid carcinoma. J Thorac Dis 2017;9:4758-66.

20. Liu H, Yan F, Pan Z, Lin X, Luo X, Shi C, Chen X, Wang $\mathrm{B}$, Zhang H. Evaluation of dual energy spectral CT in differentiating metastatic from non-metastatic lymph nodes in rectal cancer: Initial experience. Eur J Radiol 
2015;84:228-34.

21. Padera TP, Stoll BR, Tooredman JB, Capen D, Tomaso ED, Jain RK. Pathology: cancer cells compress intratumour vessels. Nature 2004;427:695.

22. Aki R, Amoh Y, Bouvet M, Katsuoka K, Hoffman RM. Color coded fluorescence imaging of lymph-node metastasis, angiogenesis, and its drug-induced inhibition. J Cell Biochem 2014;115:457-63.

23. Zhang Y, Cheng J, Hua X, Yu M, Xu C, Zhang F, $\mathrm{Xu}$ J, Wu H. Can Spectral CT Imaging Improve the Differentiation between Malignant and Benign Solitary Pulmonary Nodules? PLoS One 2016;11:e0147537.

24. Dong D, Xiao LH, Ye SP. A Preliminary Study of Dual Source CT Dual Energy Imaging of Lung Cancer Lymph Node Metastasis. J Clin Radiol 2016;35:1027-31.

25. Iwano S, Koike W, Matsuo K, Kitano M, Kawakami K, Okada T, Naganawa S. Correlation between dynamic CT findings and pathological prognostic factors of small lung adenocarcinoma. Cancer Imaging 2012;12:187-93.

26. Huber AT, Schuster F, Ebner L, BütikoferY, Ott D, Leidolt L, Jöres A, Montani M, Heverhagen J, Christe A. Hepato cellular Carcinoma Screening With Computed

Cite this article as: Gao L, Lu X, Wen Q, Hou Y. Added value of spectral parameters for the assessment of lymph node metastasis of lung cancer with dual-layer spectral detector computed tomography. Quant Imaging Med Surg 2021;11(6):2622-2633. doi: 10.21037/qims-20-1045
Tomography Using the Arterial Enhancement Fraction With Radiologic-Pathologic Correlation. Invest Radiol 2016;51:25-32.

27. Oda S, Nakaura T, Utsunomiya D, Funama Y, Taguchi N, Imuta M, Nagayama Y, Yamashita Y. Clinical potential of retrospective on-demand spectral analysis using duallayer spectral detector-computed tomography in ischemia complicating small-bowel obstruction. Emerg Radiol 2017;24:431-4.

28. Rao YY, Yang W J, Bo L. Dual energy CT scanning to evaluate the mediastinal lymph node metastases in lung cancer: Correlation of weighted iodine concentration and mediastinal lymph node metastases. Radiol Pract 2013;28:759-62.

29. Chong S, Lee KS, Chung MJ, Han J, Kwon OJ, Kim TS. Neuro-endocrine Tumors of the Lung: Clinical, Pathologic, and Imaging Findings. Radiographics 2006;26:41-57.

30. Warren WH, Gould VE. Neuroendocrine tumors of the broncho pulmonary tract: a reappraisal of their classification after 20 years. Surg Clin North Am 2002;82:525-40. 